\title{
Riesgo sísmico, geotécnico y estructural. El caso de estudio de los edificios San Jerónimo de Yuste en Bogotá
}

\author{
Seismic, geotechnical and structural risk. The Case Study of San Jerónimo \\ de Yuste Buildings in Bogotá
}

J. M. Medina $^{(*)}$, C. Villate ${ }^{(*)}$, B. Caicedo ${ }^{(*)}$, L. Yamin ${ }^{(*)}$, J. F. Correal ${ }^{(*)}$, N. Estrada ${ }^{(*)}$, J. R. Rincón $^{(*)}$

RESUMEN

El conjunto residencial San Jerónimo de Yuste se encuentra ubicado en Bogotá, en un terreno inclinado en el pie de monte de los cerros orientales de la ciudad. Fue construido entre 2008 y 2011, como parte de un proyecto de urbanización promovido por la caja de compensación familiar Compensar. Durante mayo y junio de 2013 el terreno presentó desplazamientos tanto verticales como horizontales que generaron daños visibles en las estructuras y motivaron la evacuación de dos de las seis torres que conforman el conjunto. El presente trabajo presenta los resultados de la investigación que llevaron a cabo los grupos de investigación de las facultades de Arquitectura e Ingeniería de la Universidad de los Andes para determinar las causas que condujeron al evento de desplazamiento de la estructura debidas al movimiento del terreno, estimar el coeficiente de seguridad actual y proponer intervenciones geotécnicas y estructurales que permitan recuperar la edificación.

Palabras clave: vulnerabilidad estructural, asentamiento diferencial, patología constructiva, rehabilitación de edificios.

\section{ABSTRACT}

San Jerónimo de Yuste buildings are located in Bogotá, on sloping ground, in the foothills of the city. These buildings were built between 2008 and 2011 by the company BIO Construcciones, as part of a broader development project promoted by the family compensation fund Compensar. During the months of May and June 2013 the slope suffered both vertical and horizontal displacements that visibly damaged two of the buildings, which were then evacuated. This paper presents the studies conducted by the Architecture and Engineering Schools at Universidad de los Andes, in order to determine the causes that led to movement, estimate the safety factor and propose interventions at the geotechnical and structural levels which would allow the recovery of the affected buildings.

Keywords: structural vulnerability, differential movement, settlements, building pathology, building rehabilitation.

(*) Universidad de los Andes, Colombia.

Persona de contacto/Corresponding author:jm.medinad@uniandes.edu.co (J. M. Medina)

ORCID: http://orcid.org/oooo-0002-1065-1494 (J. M. Medina); http://orcid.org/oooo-0002-3097-2009 (C. Villate);

http://orcid.org/oooo-0003-4344-0914 (B. Caicedo); http://orcid.org/oooo-0002-6533-5480 (L. Yamin);

http://orcid.org/oooo-0002-6904-0081 (J. F. Correal); http://orcid.org/oooo-0003-4941-6253 (N. Estrada);

http://orcid.org/oooo-0002-8340-4718 (J. R. Rincón)

Cómo citar este artículo/Citation: J. M. Medina; C. Villate; B. Caicedo; L. Yamin; J. F. Correal; N. Estrada; J. R. Rincón. (2018). Riesgo sísmico, geotécnico y estructural. El caso de estudio de los edificios San Jerónimo de Yuste en Bogotá. Informes de la Construcción, 70(550): e251. https://doi.org/10.3989/ic.16.131

Copyright: ( 2018 CSIC. Este es un artículo de acceso abierto distribuido bajo los términos de la licencia de uso y distribución Creative Commons Reconocimiento 4.0 Internacional (CC BY 4.0). 


\section{INTRODUCCIÓN}

Este artículo presenta los resultados derivados de la investigación que se produjo mediante un estudio de caso, a través de un análisis transversal que va desde lo arquitectónico a lo geotécnico y estructural de un conjunto de viviendas sometidas a un desplazamiento no esperado de su posición inicial. Se trata del conjunto residencial San Jerónimo de Yuste, ubicado en la calle 16 sur, número 18-50, en la Localidad de San Cristóbal, en los cerros orientales de la ciudad de Bogotá, Colombia.

Durante mayo de 2013 se observaron desplazamientos horizontales y verticales relativos de hasta 22,5 centímetros en dos de las torres y en el parqueadero, lo que sugería la existencia de un fenómeno de inestabilidad en el terreno. Para octubre de 2013, por solicitud del Instituto Distrital de Gestión de Riesgos y Cambio Climático (Idiger), se evacuó como medida preventiva una de las torres (la 3). En abril de 2014, Compensar, entidad promotora del proyecto, contacta a la Universidad de los Andes para llevar a cabo una investigación profunda del caso y generar una propuesta técnica que permitiera determinar las causas del fenómeno geotécnico, así como los niveles de seguridad normativos de la edificación y del terreno, todo ello encaminado a definir la habitabilidad del proyecto y a sugerir las posibles medidas de rehabilitación y control que permitieran alcanzar el grado de seguridad exigido por el Reglamento Colombiano de Construcción Sismo Resistente NSR-10 (2010).

\section{CONDICIONES GEOTÉCNICAS Y SÍSMICAS DE BOGOTÁ Y NORMATIVA DE REFERENCIA}

Las edificaciones objeto de estudio se encuentran en Bogotá, Colombia, en una zona de amenaza sísmica intermedia. Están ubicadas en el piedemonte de los cerros orientales que flanquean la ciudad. Estos cerros están compuestos principalmente por rocas sedimentarias clásticas y rocas metamórficas (1) con un bajo grado de metamorfismo (2). Los depósitos sedimentarios que dieron origen a estas rocas son principalmente arcillosos, aunque es posible encontrar algunos horizontes compuestos por materiales gruesos (i.e., arenas) (3) . En consecuencia, en el piedemonte de los cerros orientales bogotanos es frecuente encontrar suelos arcillosos, suelos residuales y coluviones, estos últimos ubicados en los principales causes de la red de drenaje compuesta por arroyos de montaña. En el sitio del proyecto, los depósitos de piedemonte son principalmente arcillosos. De hecho, en el pasado en el lugar existió una mina de arcilla, que se utilizaba para la fabricación de ladrillos y tejas de barro. Estos suelos, por sus características de resistencia y deformabilidad, son susceptibles a los deslizamientos y a otros fenómenos de remoción en masa (4). Por la naturaleza del terreno, son comunes los fenómenos de reptación, con velocidades de desplazamiento altamente correlacionadas con el régimen de lluvias en la ciudad.

La combinación de ambas amenazas naturales hace que la construcción en este lugar singular de la geografía bogotana requiera que se les preste mayor atención a los aspectos geotécnicos y estructurales. Por lo tanto, el proyecto de investigación comprende dos grandes áreas de análisis: el riesgo geotécnico y el riesgo estructural, y ambos, analizados desde la óptica arquitectónica, buscan determinar el estado real de los edificios construidos.

Luego de analizar la normativa vigente en Colombia en 2008, cuando se construyó la edificación, se evidencia que aún regía
Reglamento Colombiano de Construcción Sismo Resistente NSR-98 (5). Esta comprendía todos los requisitos para la realización de los estudios de suelo, evaluación de la amenaza sísmica, diseño de cimentaciones, diseño estructural y demás para la configuración del proyecto.

De este modo, se verificó con base en dicha normativa que esta se hubiera cumplido para los diseños originales de la construcción. Sin embargo, la revisión de la vulnerabilidad geotécnica y estructural requiere utilizar el reglamento más reciente a la fecha del estudio. Para este caso, los estudios y posteriores conceptos generados se basan en el Reglamento Colombiano de Construcción Sismo Resistente NSR-10. Este documento rige para edificaciones construidas a partir de 2010 y comprende diferentes títulos (6) para tener en cuenta para el análisis tanto de las edificaciones nuevas como de las existentes.

Igualmente, se usaron referencias de casos similares o investigaciones preliminares, como el caso de la investigación de las causas del colapso del Edificio Space (7) y de casos como el de la rehabilitación singular de edificios de viviendas en la barriada del Parque Alcosa (8), para la evaluación de las posibles causas y para la determinación de las recomendaciones respecto a la habitabilidad y obras de reforzamiento requeridas para un nivel de seguridad de operación óptimo del edificio.

\section{OBJETIVO}

El objetivo fundamental de la investigación es determinar un diagnóstico de las causas principales de los desplazamientos ocurridos en el edificio San Jerónimo de Yuste y de las afectaciones estructurales observadas. Con base en esto se pretende establecer el nivel de riesgo de los taludes y del edificio para el estado actual ante cargas gravitacionales y sísmicas.

\subsection{Hipótesis}

Los desplazamientos ocurridos en los edificios, sucedieron sin la presencia de cargas externas por eventos naturales (sismos, deslizamientos, etc.) y/o inducidas (excavaciones contiguas a la edificación). Esto diferencia este caso de estudio de aquellos en los cuales determinar las causas principales requiere la investigación de las condiciones de las estructuras adyacentes y de la estructura bajo revisión (Anastasopoulos, 2013) (9).

La hipótesis inicial del equipo investigador, por tanto, era que un análisis cruzado de los estudios de laboratorio, las prospecciones geotécnicas, los análisis realizados sobre los diseños estructurales y las simulaciones sísmicas, todos ellos coordinados y adaptados a la edificación existente, permitirían determinar el coeficiente de seguridad a la luz de la norma NSR-10 y, en definitiva, a establecer si los edificios eran seguros para su ocupación y uso o si requerían alguna intervención para lograr esta condición.

\section{EL EDIFICIO}

El conjunto arquitectónico está conformado por dos bloques de vivienda que se disponen de manera oblicua en torno a un espacio de estacionamiento central. Este sirve como punto de acceso al conjunto y salva la diferencia de nivel de 6 metros que se presenta entre ambos bloques, como se observa en la figura 1. 


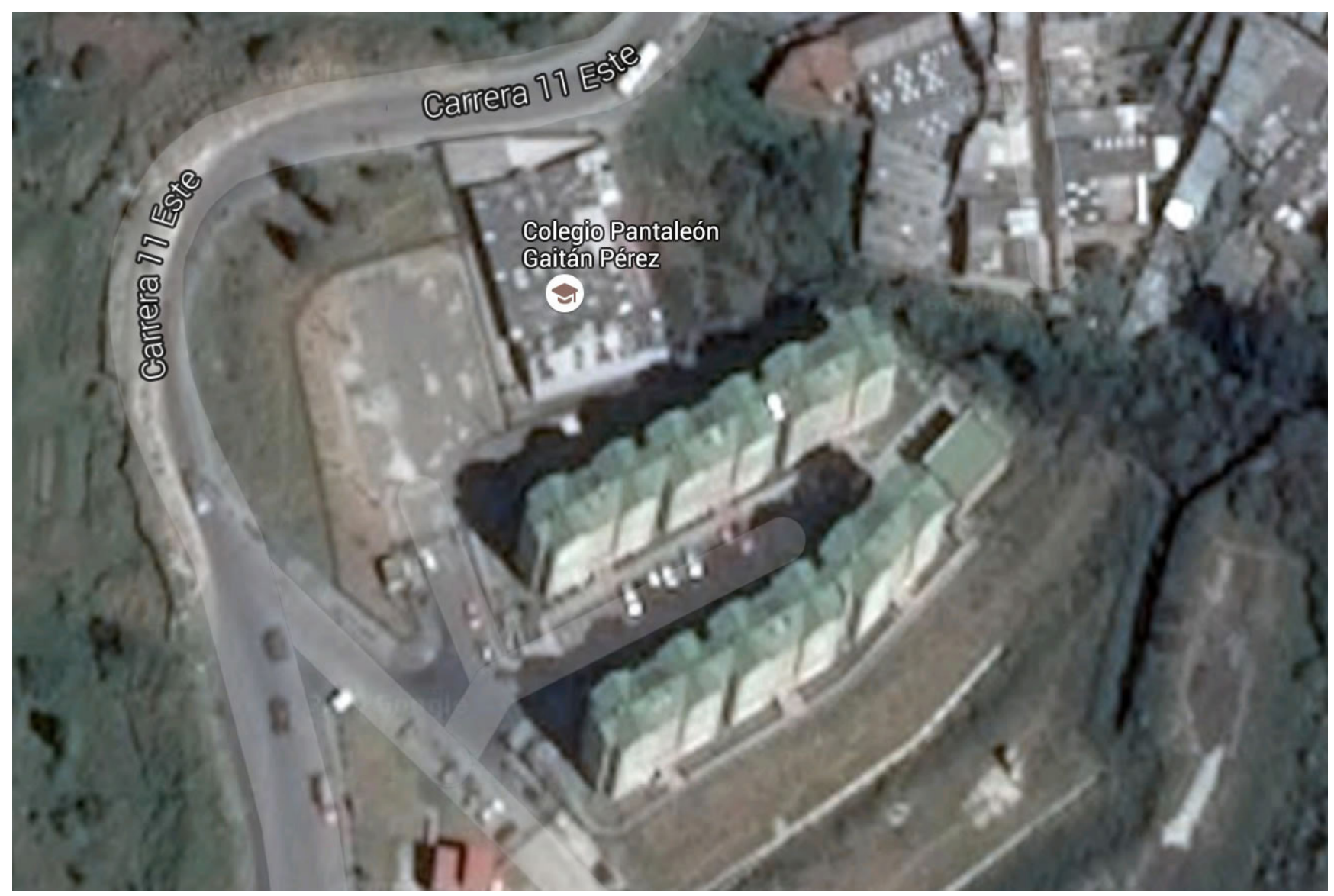

Figura 1. Conjunto arquitectónico bajo análisis. Fuente: fotografía reproducida con la autorización de Edifica Colombia, S. A.

Respecto al terreno, los bloques están ubicados de manera que su sección longitudinal (norte-sur) sea perpendicular a la inclinación del terreno (oriente-occidente). Con una configuración simétrica, los bloques reúnen cada uno tres torres de vivienda de ocho pisos, las cuales se encuentran dilatadas entre sí $\mathrm{y}$ funcionan de manera independiente. Cada una de las torres alberga un total de 28 apartamentos. Dada la inclinación del terreno, las torres tienen dos de sus pisos bajo el nivel del área de estacionamiento, lo que produce que en estos solo sea habitable la mitad de la edificación y que el acceso a las torres del lado más bajo se produzca del lado del estacionamiento y por el tercer nivel de la torre (figura 2). Es en la torre 3, ubicada en la parte más baja y en el extremo noroccidental del terreno, donde se presenta el fenómeno de desplazamiento.

Las seis torres del conjunto presentan la misma configuración simétrica que, en términos generales, es la más común para la vivienda social de iniciativa privada que se desarrolla en Colombia: un núcleo central que contiene la escalera junto con los puntos de servicio y alrededor de este se disponen cuatro apartamentos, dos de cada lado, con las mismas dimensiones. La distribución de los apartamentos es idéntica y atiende los requerimientos del Ministerio de Vivienda: un salón comedor, cocina, un baño, una zona de ropas y dos habitaciones con un área entre los $45 \mathrm{y}$ los 50 metros cuadrados.

En cuanto a la configuración estructural, está hecha por muros de carga en concreto reforzado de 10 centímetros de espesor distribuidos en las dos direcciones de la edificación, los cuales van de cimentación a cubierta (22,16 metros). Las placas de entrepiso macizas en concreto de 10 centímetros, de espesor con vigas de amarre entre muros. En algunas zo-

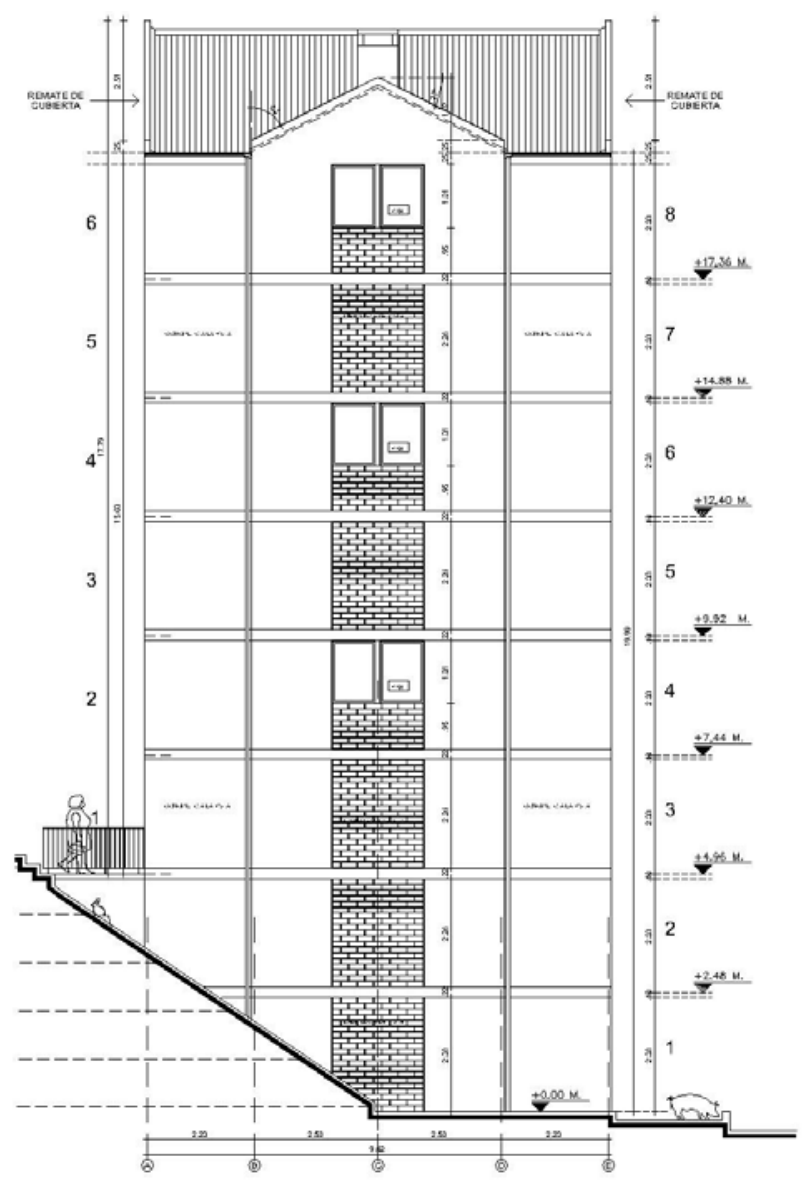

Figura 2. Fachada lateral de la edificación según planos arquitectónicos. 
nas se aprovecha el antepecho de las ventanas para lograr una mayor sección en las vigas. Algunos muros divisorios y antepechos se construyeron en bloques de arcilla.

\section{METODOLOGÍA}

\subsection{Antecedentes}

Durante mayo de 2013, previo a los estudios aquí referidos, se observaron desplazamientos en las torres 3 y 4 y en el parqueadero 7, que sugerían la existencia de un fenómeno de inestabilidad geotécnica. Los desplazamientos encontrados pueden reconocerse como movimientos mayores a los considerados como admisibles en una edificación (Walhls, 1981) (10). En los meses siguientes, a raíz de estos desplazamientos, Compensar contrató una serie de estudios (i.e., monitoreos GPS y topográficos, exploraciones geofísicas y análisis geotécnicos) encaminados a caracterizar el proceso y a identificar diferentes estrategias de estabilización. En la figura 3 se muestra una planta del proyecto, y en ella se indican los desplazamientos horizontales observados en varios de los puntos monitoreados, así como el alineamiento del deslizamiento sugerido por estos desplazamientos y las grietas observadas en campo.

\subsection{Metodología del estudio de caso}

En 2015, y tras el encargo a la Universidad de los Andes del análisis integral del caso, los estudios del proyecto se dividieron en dos frentes de trabajo: vulnerabilidad y geotecnia, junto a una coordinación de apoyo por parte de la Facultad de Arquitectura. El equipo de vulnerabilidad se encargó de eva- luar el estado estructural y el grado de vulnerabilidad frente a cargas gravitacionales y sísmicas; mientras que el equipo de geotecnia se encargó de determinar la estabilidad del terreno, así como de identificar los mecanismos de falla presentes para de ese modo poder recomendar la forma en que debía intervenirse la ladera para resolver el problema existente.

\section{ANÁLISIS DEL EQUIPO DE GEOTECNIA}

Para llevar a cabo el estudio, el equipo de geotecnia de la universidad empezó por revisar los estudios previos. Luego los sondeos y los muestreos realizados por Edifica Colombia Ltda., empresa colaboradora de la investigación, se utilizaron para proponer un perfil geotécnico de la ladera en varios alineamientos. Después, y a partir de este perfil geotécnico, se agruparon los suelos observados en varias unidades geotécnicas y se seleccionaron varias muestras que luego se probaron en el laboratorio, a fin de evaluar las propiedades mecánicas de los diferentes estratos de suelo. Específicamente, se condujeron ensayos de gravedad específica (11) , peso unitario (12), humedad natural (13) según su normativa de regulación (14), límites de Atterberg (15) y corte directo (16) . Estos ensayos se condujeron con el fin de determinar el contenido de agua en los estratos arcillosos, lo que permite deducir su susceptibilidad a fenómenos de reptación, y la resistencia al corte de las unidades geológicas atravesadas por la superficie de falla. Finalmente, el perfil geotécnico de la ladera y las propiedades mecánicas de los materiales se usaron para conducir una serie de simulaciones numéricas encaminadas a estimar el factor de seguridad de la ladera en diferentes etapas del proyecto y así poder analizar el efecto de eventuales fluctuaciones del nivel freático en la estabilidad del sistema.

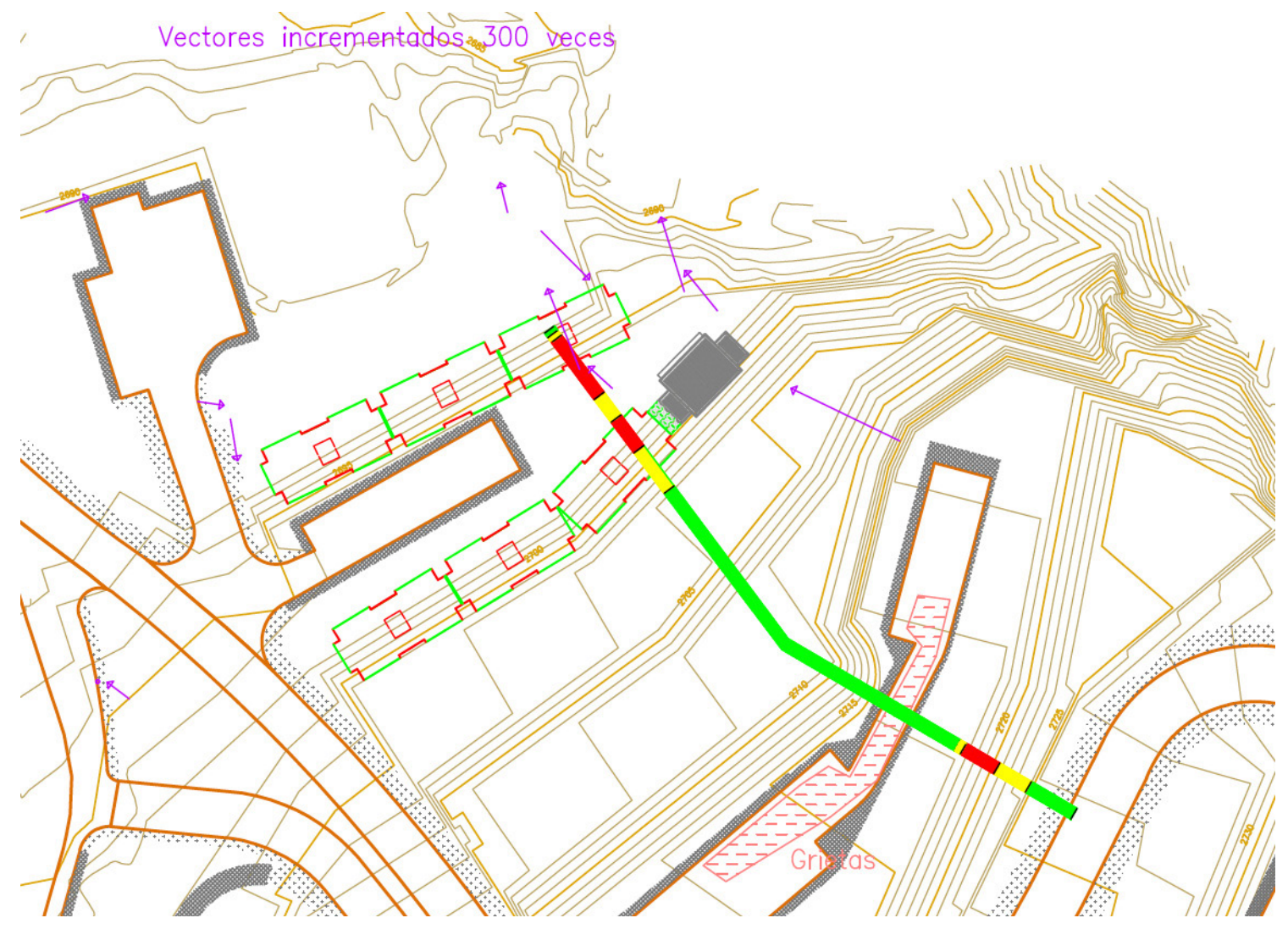

Figura 3. Alineamiento del deslizamiento (línea gruesa, de color verde, amarillo y rojo) sugerido por los desplazamientos (vectores morados, con magnitud exagerada 300 veces) y las grietas observadas en campo. 


\subsection{Análisis del movimiento y riesgos geotécnicos futuros}

A partir de las pruebas de laboratorio y perfil geotécnico, al igual que de la revisión de los estudios previos, se pudo verificar que la composición del suelo de la ladera tiene concentraciones arcillosas con altos contenidos de agua y baja resistencia al corte. Muy probablemente, su baja resistencia al corte fue la responsable de las inestabilidades observadas en 2013. Adicionalmente, estos estudios sugirieron que el factor detonante de la inestabilidad fue el agua subterránea.

En la figura 4 se muestra una planta del proyecto, y en ella se indican los puntos en los que Edifica Colombia Ltda. realizó sus sondeos y muestreos. A partir de la información recolectada en esta campaña experimental, se propuso el perfil geotécnico mostrado en la figura 5. En la tabla 1 se muestran las propiedades de resistencia al corte de las diferentes unidades que componen el perfil geotécnico.

\subsection{Modelos de elementos finitos}

A partir del perfil geotécnico de la ladera y de las propiedades mecánicas del material, con el propósito de identificar los mecanismos de falla más probables y estimar su factor de se- guridad, se modeló el sistema en un programa de elementos finitos (Plaxis 2D). Usando este programa, fue posible estimar el factor de seguridad de la ladera mediante la técnica de la reducción de parámetros (17). Para estos análisis se usaron las propiedades mínimas evaluadas en el laboratorio (18), ubicando el análisis del lado de la seguridad (19). Se decidió usar la técnica de la reducción de parámetros porque permite obtener resultados similares a los que se obtienen mediante análisis por equilibrio límite, con la ventaja de no prescribir la ubicación y la geometría de la superficie de falla. Adicionalmente, al ser implementada en un código de elementos finitos como Plaxis 2D, permite conducir simultáneamente análisis de deformaciones y de estabilidad, problemas que normalmente se abordan mediante herramientas diferentes en la ingeniería geotécnica.

En la figura 6 se muestran los resultados de dos de los modelos con elementos finitos correspondientes a tres estados del proyecto. El primero de estos tiene en cuenta la primera acción correctiva que se implementó, que fue abatir el nivel freático. El segundo tiene en cuenta la segunda acción correctiva, que fue la construcción de una cortina de pilotes. Finalmente, el tercer estado corresponde al sistema con el nivel freático abatido y con la cortina de pilotes construida, pero bajo el efecto de un sismo, según la metodología indicada en la NSR-10.

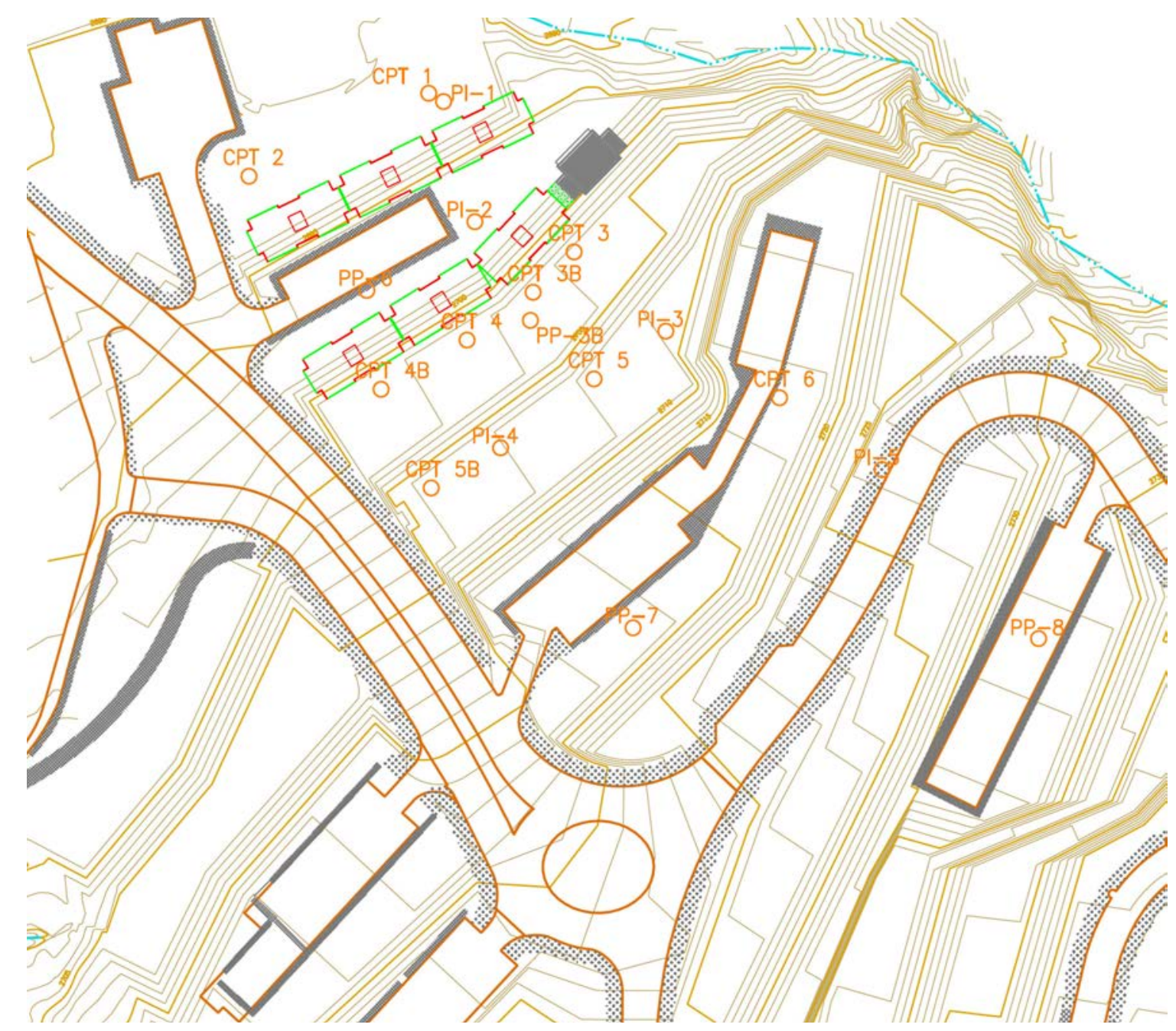

Figura 4. Sondeos realizados por Edifica Colombia Ltda. PI: perforación con inclinómetro; PP: perforación con presurómetro; CPT: sondeo con piezocono. 


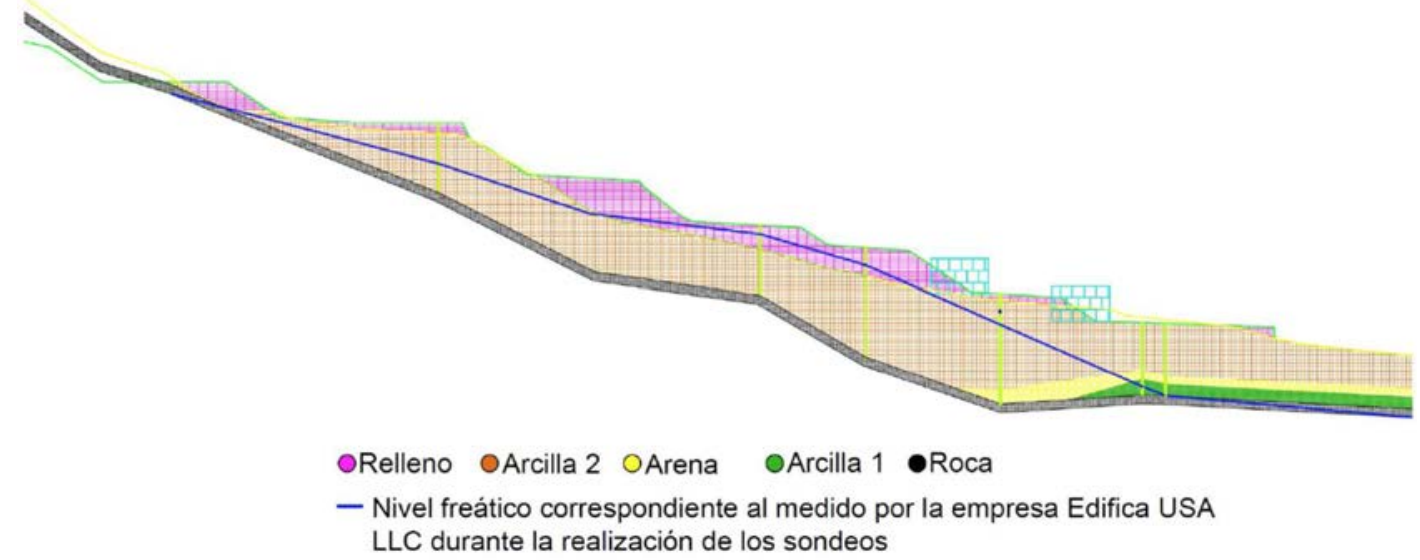

Figura 5. Perfil geotécnico correspondiente al alineamiento presentado en la figura 3.

Tabla 1. Propiedades de resistencia al corte de las unidades que componen el perfil geotécnico.

Arcilla No. 1

\begin{tabular}{c|c}
\hline$c^{\prime}$ & $0 \mathrm{kPa}$ \\
$\phi^{\prime}$ & $26^{\circ}$ \\
\hline \multicolumn{2}{c}{ Arena } \\
\hline$c^{\prime}$ & $0 \mathrm{kPa}$ \\
$\phi^{\prime}$ & $33^{\circ}$ \\
\hline
\end{tabular}

Arcilla No. 2 bajo el nivel freático

\begin{tabular}{c|c}
\hline$c_{\min }^{\prime}$ & $0 \mathrm{kPa}$ \\
$\phi_{\min }^{\prime}$ & $21^{\circ}$ \\
$c_{\max }^{\prime}$ & $4 \mathrm{kPa}$ \\
$\phi_{\max }^{\prime}$ & $28,5^{\circ}$ \\
\hline
\end{tabular}

Arcilla No. 2 sobre el nivel freático

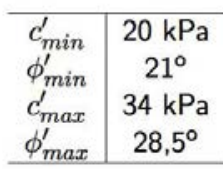

Relleno

\begin{tabular}{c|c}
\hline$c^{\prime}$ & $8 \mathrm{kPa}$ \\
$\phi^{\prime}$ & $41,1^{\circ}$ \\
\hline
\end{tabular}

En primer lugar, se observa que la instalación de los pilotes incrementa el factor de seguridad, aunque este aumento es relativamente pequeño. En segundo lugar, se observa que el factor de seguridad actual en condiciones estáticas (i.e., 1,67) es ligeramente superior al exigido en la NSR-10 (i.e., 1,5). Finalmente, se observa que el factor de seguridad en condiciones sísmicas es inferior al exigido en esta misma norma, que es igual a 1,05. Nótese que las superficies de falla críticas identificadas por la herramienta de simulación son consistentes con el alineamiento sugerido por las observaciones de campo y presentado en la figura 3 de este artículo.

\subsection{Efecto del agua en el movimiento del terreno}

Tanto en los estudios previos como en los modelos de elementos finitos realizados, es claro que la posición del nivel freático tiene un efecto mayor en la estabilidad y las deformaciones de la ladera. Por esta razón, se decidió analizar en detalle la correlación entre el agua subterránea y la estabilidad de la ladera.
Inicialmente, se decidió explorar cuantitativamente el efecto de la posición del nivel freático en el factor de seguridad de la ladera. Para hacerlo, se partió de una situación en la que se supuso el nivel freático en la superficie. Luego, se abatió gradualmente el nivel freático y para cada posición se estimó el factor de seguridad. Se consideraron el caso sin pilotes y el caso con los pilotes instalados. Los resultados de este análisis se muestran en la figura 7 .

Se observa que, para posiciones del nivel freático a menos de 2 metros de la superficie, la ladera es inestable para los dos sistemas considerados. A pesar de la simplicidad de este análisis, estos resultados son importantes, ya que, como se señala en los estudios previos, existen mediciones en campo en las cuales se registraron profundidades del nivel freático cercanas a 1 metro, medido a partir de la superficie del terreno. Por otro lado, estos resultados señalan la importancia de asegurar el buen funcionamiento de los sistemas de drenaje durante la vida útil de la estructura, ya que, si estos fallaran y el nivel freático aumentara, la inestabilidad observada en 2013 podría reactivarse.

\section{ANÁLISIS DEL EQUIPO DE INGENIERÍA ESTRUCTURAL Y VULNERABILIDAD SÍSMICA}

Los asentamientos verticales y los desplazamientos horizontales presentados por el terreno se manifestaron mediante patologías estructurales sobre el proyecto de vivienda San Jerónimo de Yuste, principalmente en la torre 3. Desde la primera aparición de los daños sobre la estructura, Compensar realizó un seguimiento topográfico para tener el registro de los desplazamientos y a su vez como sistema de monitoreo. Una vez se contactó con los revisores estructurales, el Centro de Investigaciones en Materiales y Obras Civiles (CIMOC), de la Universidad de los Andes, se definieron cuatro actividades principales como objetivo del estudio de vulnerabilidad estructural:

- Verificación del diseño estructural original para detectar si hubo fallas en el proceso de diseño.

- Análisis de vulnerabilidad sísmica en conjunto con los asentamientos diferenciales presentados.

- Análisis de las patologías y confrontación con los resultados encontrados.

- Recomendación de intervención desde el punto de vista estructural.

El presente artículo presenta la metodología y desarrollo de cada una de las actividades que sirvieron para determinar las 


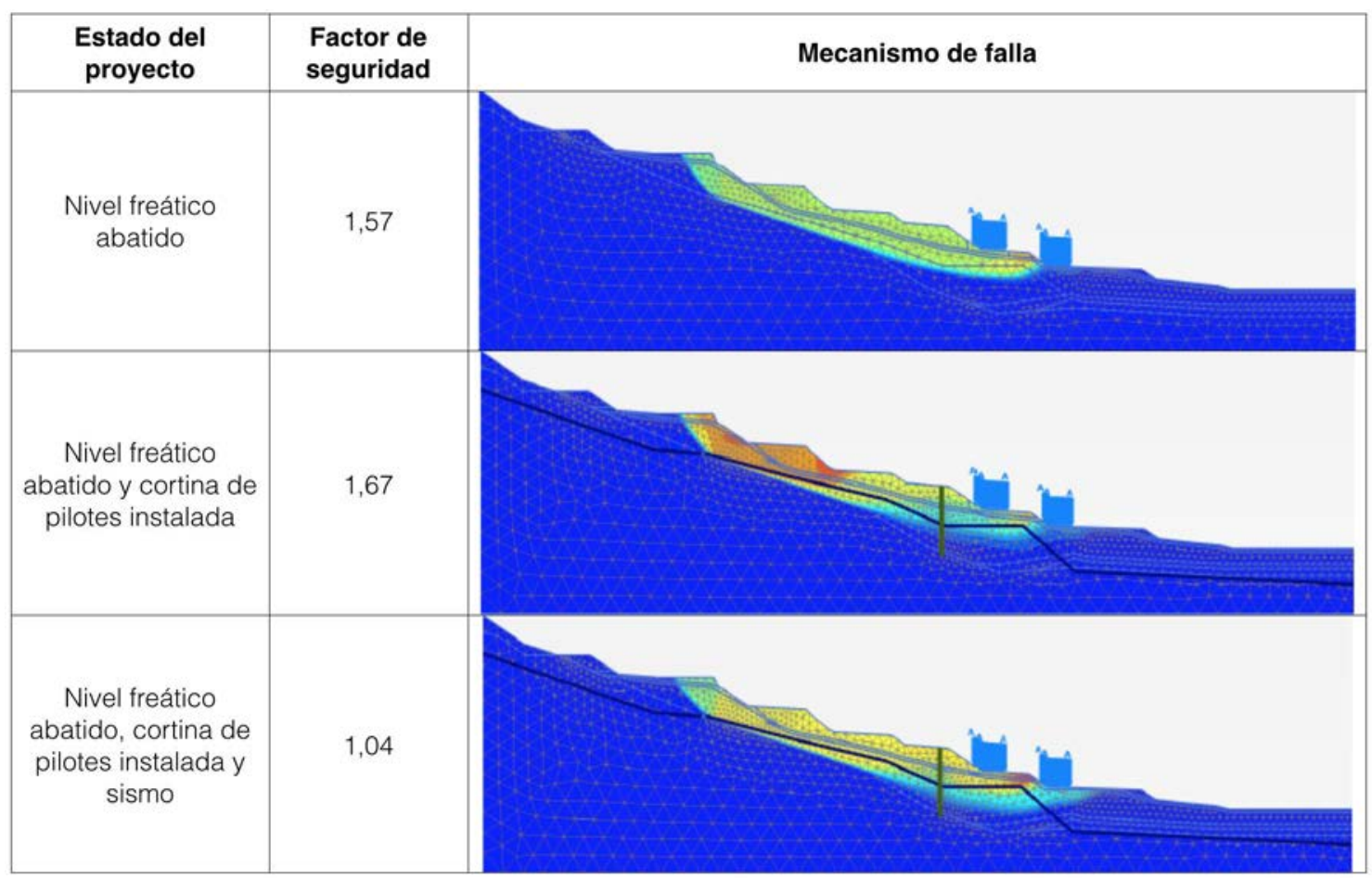

Figura 6. Resultados de los modelos de elementos finitos correspondientes a tres estados del proyecto. La escala de colores representa los desplazamientos totales de los elementos y permite identificar el mecanismo de falla analizado.

conclusiones finales de la investigación respecto a la seguridad actual de la estructura y las medidas de intervención requeridas.

\subsection{Estructuración básica de la edificación y verificación del diseño original}

La estructura básica de las torres está conformada por muros estructurales de carga en concreto reforzado en las dos direcciones principales del edificio y losas macizas de espesor constante a nivel de cada una de las placas del entrepiso con algunas vigas de amarre en el sistema de diafragma.

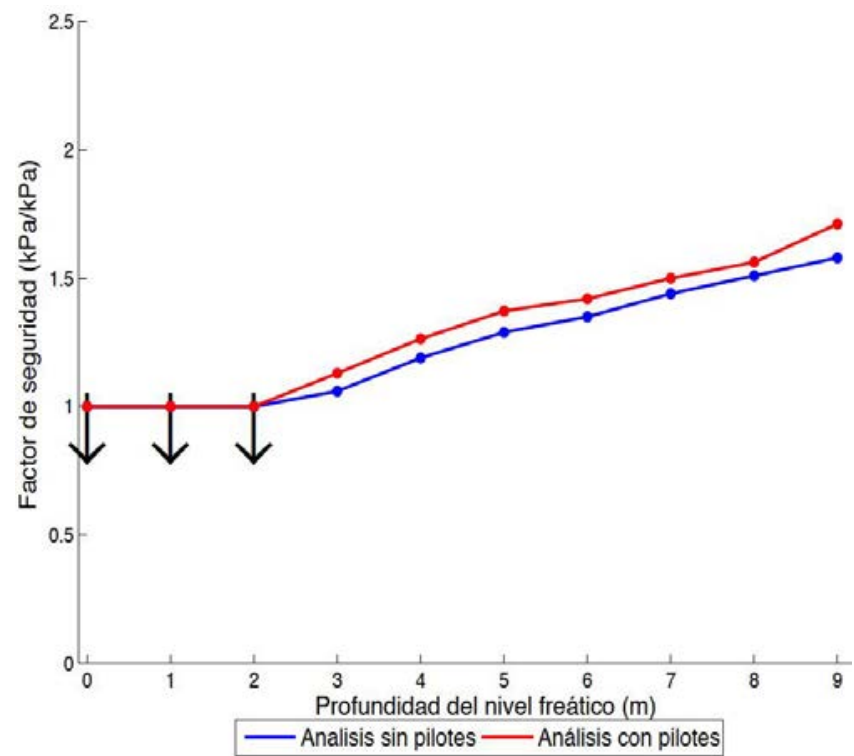

Figura 7. Sensibilidad del factor de seguridad de la ladera, en el perfil crítico, ante la variación de la posición del nivel freático.
Las edificaciones cuentan con ocho pisos, de los cuales los dos primeros niveles reducen su área en planta, dada la configuración de la ladera. Esto implica que el nivel de cimentación no sea el mismo en toda la planta de la edificación. Se presentan vigas escalonadas para la unión de elementos estructurales entre el eje de fachada y el eje central de la edificación. Las torres cuentan con una altura máxima de 22,16 metros y la altura libre (sin considerar los dos primeros niveles) desde el piso 3 hasta la cubierta de la torre es de 17,20 metros. El acceso a las edificaciones es entonces por el nivel 3 (véase figura 2).

Para la verificación del diseño original se llevó a cabo el procedimiento de evaluación de la vulnerabilidad de edificaciones existentes propuesto por la NSR-10 (Literal A.10.1.4) sumado a actividades particulares propuestas por el equipo investigador dada las implicaciones geotécnicas sobre la estructura. Dicha metodología se resume en la figura 8 y representa adicionalmente las actividades principales objeto de estudio. Bajo esta metodología se llevó a cabo un análisis del diseño original de la edificación. En dicha revisión se consideraron los requisitos impuestos por la NSR-98 para el diseño de edificaciones sismorresistentes.

Para la evaluación de la capacidad y calidad de los materiales de construcción se utilizaron dos fuentes de información: el registro de control de calidad durante la construcción y los ensayos de laboratorio a las muestras extraídas directamente de la edificación bajo estudio. Para el presente proyecto se realizó la extracción de un total de 17 núcleos de concreto sobre los elementos estructurales y de cimentación, y la verificación de la localización y el detallamiento del acero de refuerzo se realizaron mediante ensayos Ferroscan. Se encuentra que los valores de las capacidades del concreto son superiores a los valores reportados por el diseñador y que el acero de refuerzo es consistente por el presentado en los planos del diseño. 


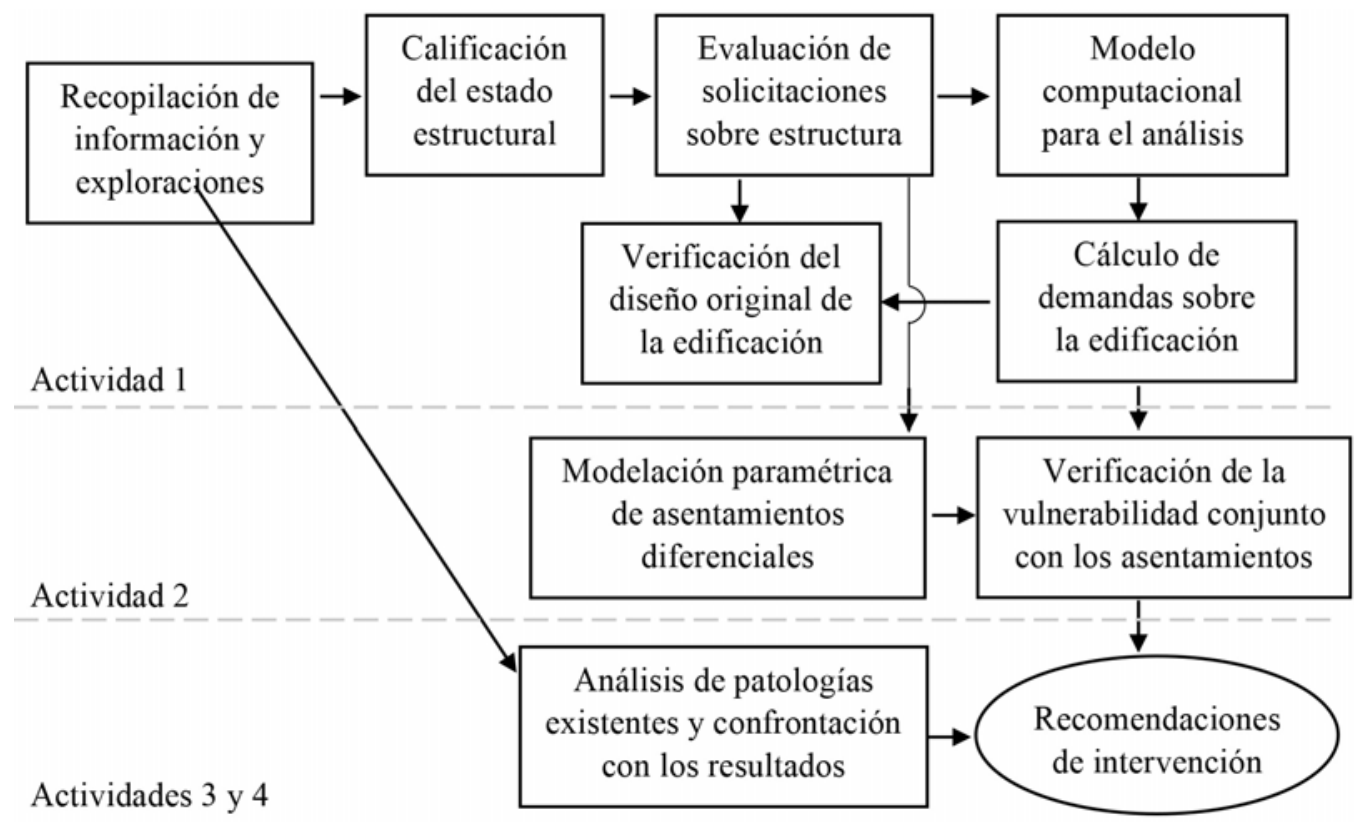

Figura 8. Esquema general de la metodología de evaluación propuesta.

Estas verificaciones permiten constatar la buena calidad de la construcción y sirven de soporte para la verificación de la seguridad de la edificación junto con los resultados obtenidos posterior al análisis computacional.

La modelación tridimensional (figura 9) tiene como propósito contar con valoraciones confiables de las demandas, en términos de desplazamientos verticales u horizontales, fuerzas y esfuerzos internos en los elementos estructurales principales para las diferentes combinaciones de carga establecidas por la NSR-98. Para el presente caso de evaluación se realizó un análisis dinámico espectral (definido en la NSR-10 literal A.5.4) considerado apropiado para el cálculo de las características relevantes de la respuesta dinámica. Esta demanda

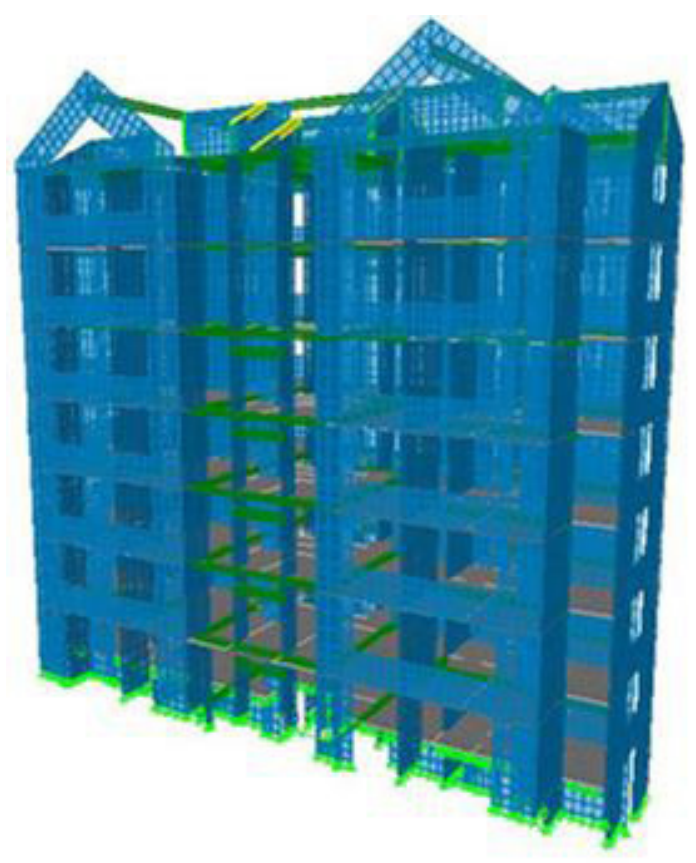

Figura 9. Modelo tridimensional realizado con elementos finitos de la estructura. obtenida es comparada con las capacidades de los elementos calculadas según las dimensiones, distribución del refuerzo y propiedades de materiales especificadas. De acuerdo con investigaciones previas, el cumplimiento de la normativa de diseño sismo resistente colombiana indica un buen comportamiento estructural de las edificaciones frente a cargas gravitacionales y sísmicas (Yamin et.al, 2014). (20)

Durante la exploración de la edificación se identificaron daños sobre las losas de entrepiso. Con el fin de verificar la posible causa de estas patologías, se llevó a cabo el análisis de deflexiones verticales en las losas típicas de entrepiso, a efectos de verificar que estas no excedan los valores límite dados por la norma. En el modelo utilizado para esta evaluación sólo se aplican las cargas muertas y vivas sin factores de sobrecarga ni inclusión de sismo. Se verifica en el presente estudio que la capacidad del suelo ante cargas gravitacionales y sísmicas, para las combinaciones de diseño, no sobrepase su capacidad correspondiente. Igualmente, se revisan a la luz de la normativa las capacidades de las vigas de cimentación y de los micropilotes existentes encargados de tomar la demanda a tensión generada por el momento de volcamiento del edificio durante eventos sísmicos. Estos últimos presentaron daños con los desplazamientos registrados.

Se encuentra posterior a los análisis descritos que la edificación cumple los requisitos de la normativa y ha sido diseñada de manera optimizada, es decir, los requisitos sí son cubiertos durante el diseño y a su vez están muy cercanos a los límites mínimos o máximos especificados. Un ejemplo de esto es la capacidad a cargas axiales de los muros estructurales. Estos requieren elementos de borde cuando los esfuerzos máximos en las fibras extremas debidas a las cargas mayoradas que resultan de las combinaciones de carga en que se incluyen los efectos sísmicos resulten superiores al 30\% de la capacidad especificada del concreto (o,3 $\mathrm{f}^{\prime} \mathrm{c}$ ). La figura 10 presenta los valores de esfuerzos de compresión encontrados para la verificación del diseño. En esta se indica que no fue necesario implementar elementos de borde en el diseño inicial y se observa un cumplimiento al límite de este requisito. 


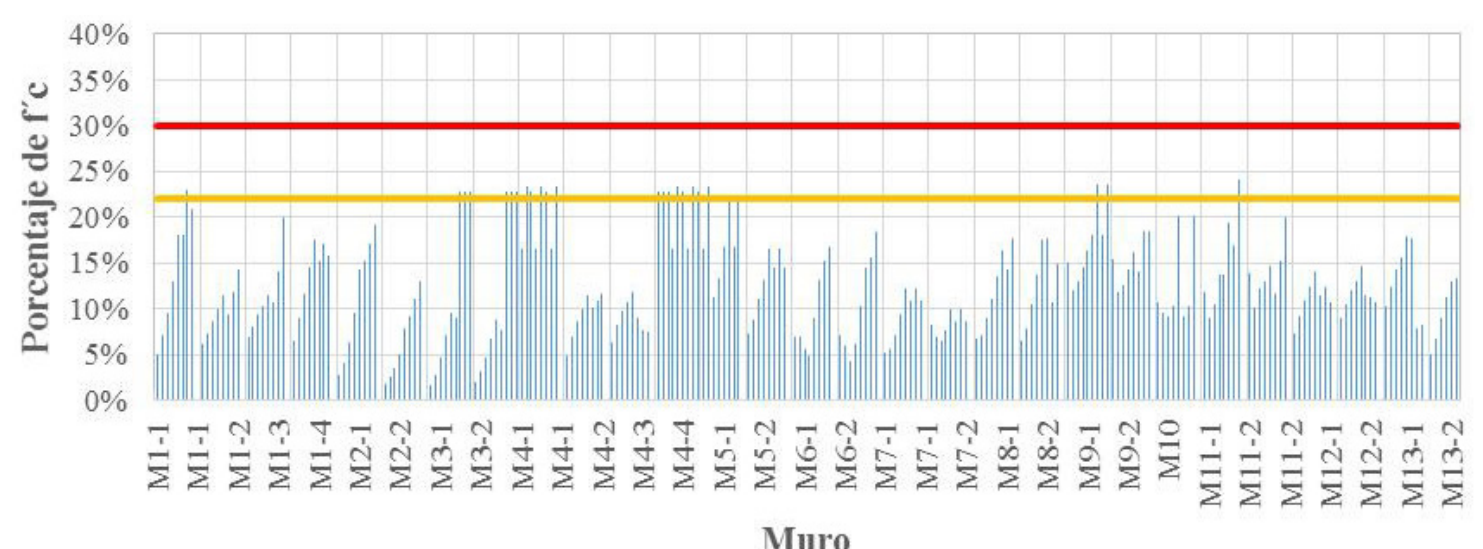

Figura 10. Esfuerzos máximos de compresión sobre muros debidos a cargas axiales y flectoras.

\subsection{Análisis de vulnerabilidad}

Para evaluar la vulnerabilidad sísmica se utilizó la normativa NSR-10 y el procedimiento descrito en la figura 8. En este caso se verificaron los índices de sobreesfuerzo (relación demanda-capacidad) en los elementos estructurales debidos a los siguientes posibles escenarios de análisis:

- Caso 1. Evaluación de vulnerabilidad para las condiciones actuales de la edificación. Se tienen en cuenta las combinaciones de carga de la NSR-10.

- Caso 2. Evaluación de vulnerabilidad para condiciones actuales de la edificación teniendo en cuenta las combinaciones de carga de la NSR-10 y los asentamientos verticales más probables en la actualidad.

- Caso 3. Análisis de sensibilidad paramétrico de asentamientos verticales diferenciales en conjunto con las demás combinaciones de carga de la NSR-10, sin incluir los casos sísmicos.

Para el análisis de estos casos se utilizó el mismo modelo tridimensional utilizado durante la revisión del diseño y se le impusieron los nuevos casos de carga debidos a los asentamientos diferenciales. Con este propósito establecido, durante los trabajos de campo se realizó el levantamiento topográfico sobre toda la edificación, principalmente registrando el nivel de cada losa aérea. Con el procesamiento de esta información se consiguió reconocer el patrón de desplazamientos laterales y verticales adoptados por la edificación. Posterior al análisis de los datos obtenidos se encuentra que la deformación de la edificación se rige por una ecuación del tipo: A $+\mathrm{BX}+\mathrm{CY}+\mathrm{DXY}$, donde su punto más bajo se encuentra en el costado sur de la fachada posterior (aproximadamente 4 centímetros).

La deformada encontrada para las placas de entrepiso es utilizada para los Casos 2 y Caso 3 de la evaluación estructural. Para el Caso 3 se realiza un análisis paramétrico de asentamientos en el cual los valores de la deformación original son aumentados en valores progresivos, a fin de identificar el nivel de asentamientos que puede poner en riesgo la construcción. La figura 11 esquematiza la situación deformada de una planta estructural para cada valor (siendo 4 centímetros el estado alcanzado en la actualidad).

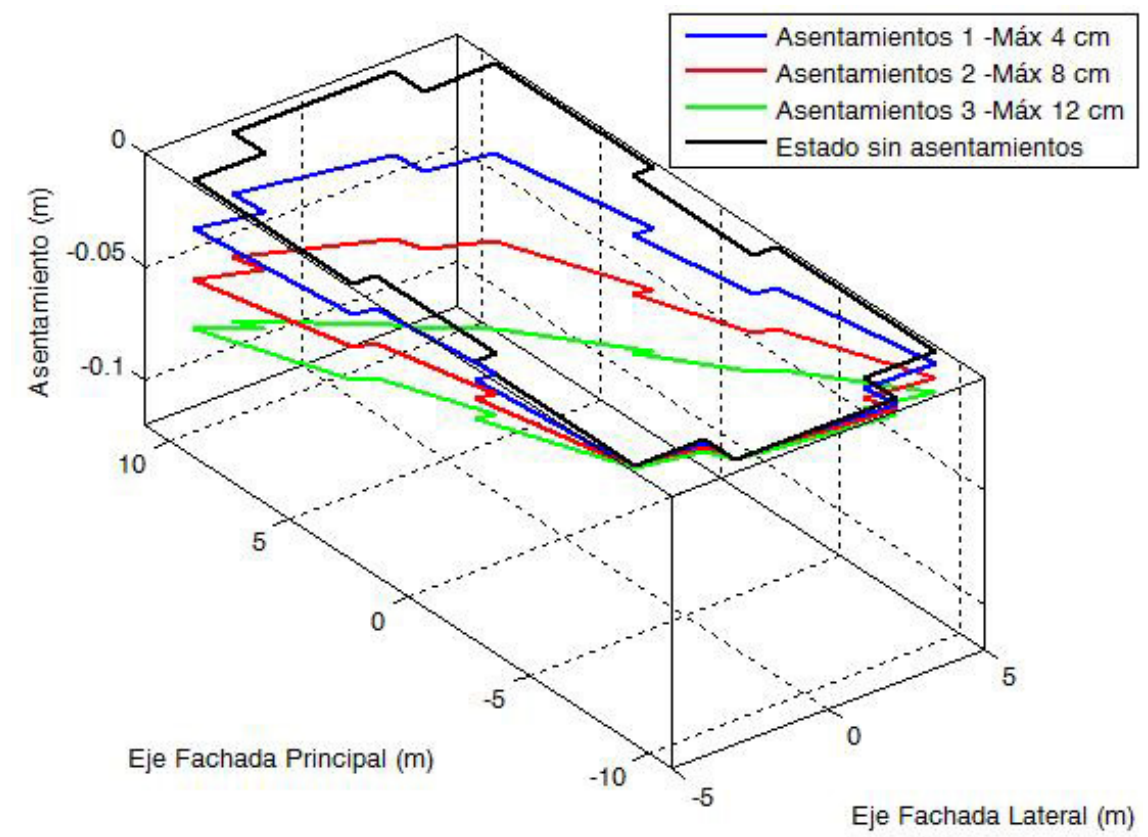

Figura 11. Esquema de asentamientos para el análisis paramétrico, asignado a la planta inferior de la edificación. 


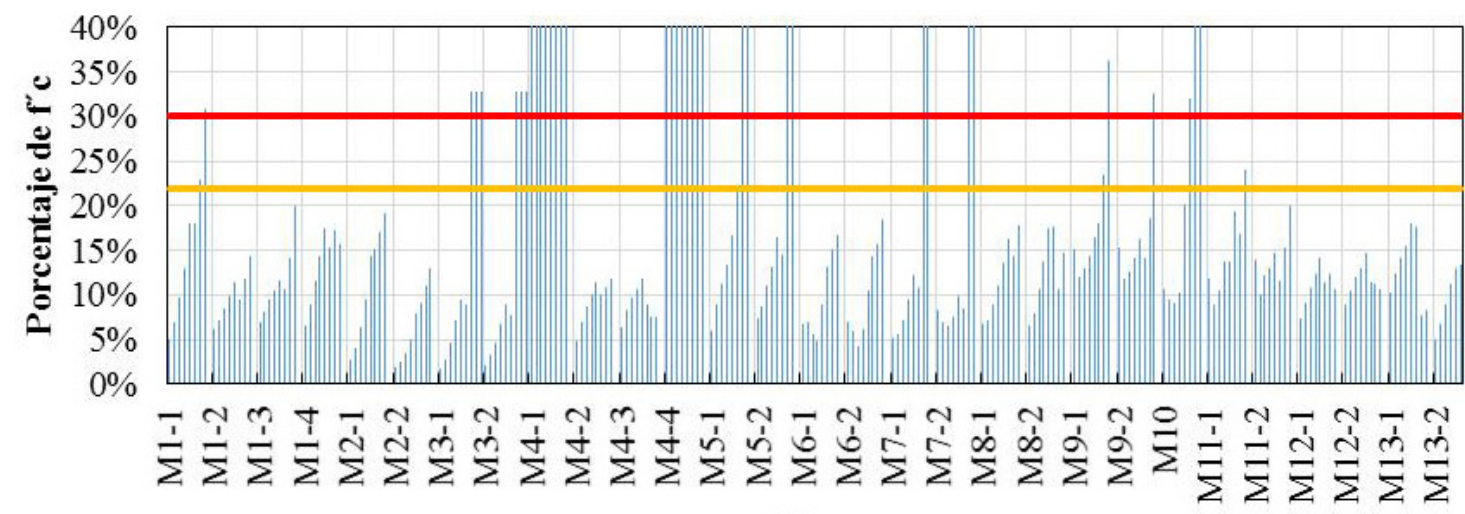

\section{Muro}

Figura 12. Esfuerzos máximos de compresión sobre muros debidos a cargas axiales y flectoras con asentamientos diferenciales.

Los resultados para el Caso 1 son similares a los encontrados en la Actividad 1 puesto que la modificación planteada sobre el modelo computacional consistió en la actualización de las cargas recomendadas por la normativa. Para el Caso 2 se determina que los asentamientos diferenciales generan un efecto negativo en los muros estructurales, puesto que generan una mayor demanda sobre los muros del primer piso. La figura 12 muestra la necesidad de incluir elementos de borde en algunos elementos dado que se sobrepasa la capacidad máxima (línea roja). Los resultados para el Caso 3, aunque no se presentan en este artículo, describen un panorama desfavorable en el caso tal que los asentamientos aumenten y el efecto de estos se pueda combinar con un evento sísmico.

\section{7•3. Análisis de las patologías y confrontación con los resultados encontrados}

Con el registro fotográfico de diferentes fechas y los informes de los conceptos estructurales suministrados por Com- pensar se levantaron los planos de daños y patologías (ver figura 13).

Con el fin de estudiar las posibles causas de las fisuras que presentan las losas del entrepiso se utiliza el modelo anterior incluyendo los asentamientos diferenciales y las cargas gravitacionales. La figura 14 esquematiza los esfuerzos inducidos por los asentamientos diferenciales, junto a las cargas verticales, considerando un valor de asentamiento diferencial de toda la estructura máximo del orden de 4 centímetros. De acuerdo a la literatura, los daños debidos a los asentamientos diferenciales están relacionados con la esbeltez de la estructura y la rigidez a cortante y flexión de los elementos principales de la estructura (Burland et.al, 1975) (21).

Se observa que el patrón de daños tomado del registro fotográfico es similar al encontrado para el modelo computacional que incluye los efectos de los asentamientos. Se considera que estos efectos pueden generarse en conjunto con la retrac-
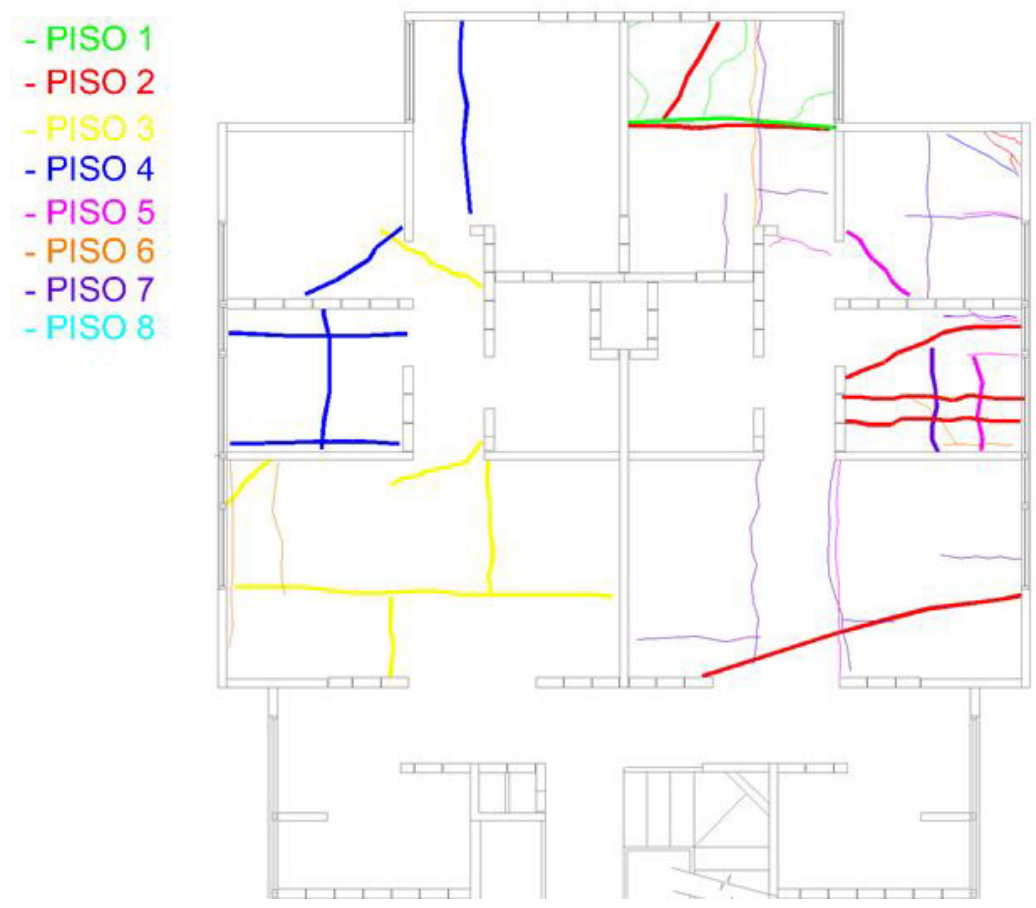

Figura 13. Fisuras con un espesor mayor a 0,5 milímetros. En cada uno de los niveles que conforman la torre bajo estudio (solo se presenta mitad de la planta). 
ción y el fraguado y la disminución de espesor de la losa en la ubicación de tuberías.

\section{CONCLUSIONES}

En el área de geotecnia, los modelos de estabilidad realizados -tanto en los estudios previos al contrato como en aquellos que hacen parte de este- indican que la condición de estabilidad de la ladera, antes de implementar las medidas de estabilización (i. e., el sistema de drenaje y la barrera de pilotes) era precaria. Este resultado teórico es consistente con los desplazamientos observados en 2013 y concuerda con el hecho de que el suelo sobre el cual se encuentra el conjunto residencial tiene niveles arcillosos con altos contenidos de agua y baja resistencia al corte. En condiciones estáticas, los modelos de elementos finitos muestran que las medidas de estabilización implementadas aumentan el factor de seguridad de la ladera hasta valores ligeramente superiores a los exigidos por la NSR-10. Bajo el efecto de un sismo, considerado mediante un análisis seudoestático, estos modelos muestran que el factor de seguridad es muy cercano al exigido por la norma. Adicionalmente, los modelos de estabilidad sugieren que el factor detonante del deslizamiento fue el agua subterránea y confirman que la posición del nivel freático tiene un efecto mayor en la estabilidad de la ladera, de tal forma que pequeñas variaciones (e. g., de uno o dos metros) del nivel freático pueden hacer que el factor de seguridad sea inferior al exigido en la norma.

Adicionalmente, se concluye que el diseño y la concepción original del edificio cumple de manera integral los requisitos aplicables de la norma NSR-98 en relación con edificaciones de muros estructurales de concreto reforzado con capacidad moderada de disipación de energía. De acuerdo con esto, se considera que el diseño original de la edificación es adecuado y se ajusta a la normativa sismorresistente aplicable.

Los asentamientos diferenciales registrados en la edificación, generan una disminución sensible en los factores de seguridad de algunos de sus muros estructurales principales. Al considerar asentamientos diferenciales máximos en dirección longitudinal del edificio en el orden de los 40 milímetros, se encuentra que los factores de seguridad para algunos de los muros de los primeros dos pisos de la edificación están por debajo de los niveles mínimos establecidos por la NSR10. Las losas de entrepiso cumplen con los requerimientos aplicables de la NSR-10: están en capacidad de resistir las demandas impuestas por las combinaciones de carga de diseño y cuentan con la rigidez mínima para limitar el daño en elementos no estructurales. Sin embargo, como consecuencia principal de su poco espesor, estas presentan una serie de fisuras, posiblemente relacionadas con alguno de los siguientes factores: retracción de fraguado del concreto durante la fase constructiva, efecto directo de las cargas muertas y vivas actuantes, sobresfuerzos inducidos por los asentamientos diferenciales registrados en el edificio, zonas de debilidad en la placa debido a la presencia de tuberías y ductos embebidos o una combinación de los anteriores. Adicionalmente, los análisis indican que las vigas de soporte y de amarre en la cimentación son adecuadas para resistir las cargas actuantes, según las consideraciones de diseño, teniendo en cuenta además los esfuerzos inducidos por los asentamientos diferenciales. Los micropilotes de cimentación definidos en los planos de diseño son adecuados para resistir las fuerzas de volcamiento de acuerdo con las condiciones de diseño originales del edificio. Las inspecciones realizadas en campo indican que estos sufrieron algunos daños, sobre todo en la parte superior en la zona de contacto con la estructura.

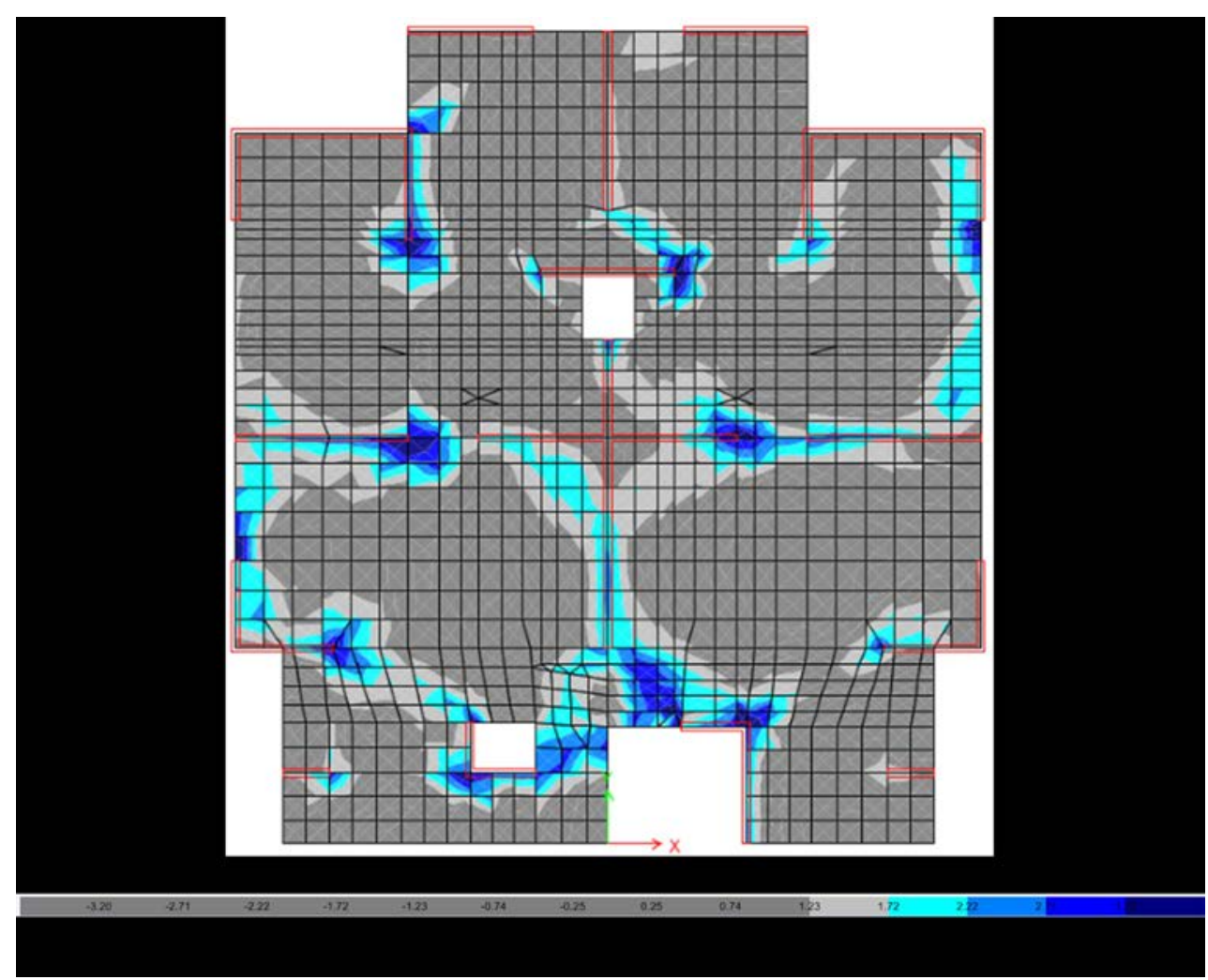

Figura 14. Esfuerzos máximos de tensión en la losa del entrepiso por cargas verticales y asentamientos diferenciales (escala de valores en MPa, solo se presenta mitad de la planta). 


\section{PROPUESTA DE INTERVENCIÓN}

Desde el punto de vista geotécnico, el estudio culmina recomendando las siguientes acciones:

1. Evaluar la posibilidad de implementar medidas de estabilización adicionales que aumenten aún más el factor de seguridad de la ladera.

2. Continuar el seguimiento de la instrumentación instalada, y eventualmente completarla con la instalación de más piezómetros.

3. Implementar un sistema de monitoreo que permita detectar a tiempo condiciones que requieran alguna intervención.

4. Implementar un plan de mantenimiento de los sistemas de estabilización que asegure su buen funcionamiento durante la vida útil de las estructuras afectadas.

En relación con el primer punto, es importante señalar que los modelos numéricos realizados en el marco de este estudio sugieren que las medidas de estabilización más eficaces son aquellas encaminadas a abatir el nivel freático. El segundo punto es esencial, puesto que el seguimiento de la instrumentación instalada es justamente el insumo del que debe alimentarse el sistema de alerta mencionado en el tercer punto. Por último, el cuarto punto es de suma importancia, ya que -como se mostró mediante los análisis de elementos finitos y como puede deducirse de los estudios previos- si los sistemas de estabilización (específicamente los sistemas de drenaje) dejaran de funcionar y el nivel freático aumentara, podrían iniciarse deslizamientos que afectarían la integridad de las torres que componen el conjunto residencial.

Posterior a la verificación del diseño, la evaluación de la vulnerabilidad estructural ante eventos sísmicos en conjunto con los asentamientos diferenciales registrados y el análisis de patologías, la edificación debe someterse a una serie de trabajos que incluyen los siguientes:

- Reparación de la totalidad de las fisuras que presenten espesores superiores a los 0,5 milímetros y que tengan patrones claramente definidos o que atraviesen elementos estructurales importantes.

- Inspección detallada, escarificación y revisión del concreto en la parte superior de los pilotes y en la zona de contacto con las vigas de amarre.

- Reforzamiento de los muros estructurales críticos en los pisos 1 y 2 mediante elementos de concreto reforzado, a manera de elementos de borde que generen confinamiento y resistencia complementaria.

\section{REFERENCIAS}

(1) SGC (2015). Mapa geológico de Colombia. Compilando la geología de Colombia: Una visión a 2015. https://www2.sgc. gov.co/ProgramasDeInvestigacion/Geociencias/Paginas/MapaGeologico.aspx.

(2) Gómez, J., Nivia, Á., Montes, N. E., Diederix, H., Almanza, M. F., Alcárcel, F. A. \& Madrid, C. A. 2015. Explanatory notes: Geological Map of Colombia. In: Gómez, J. \& Almanza, M.F. (Editors), Compilando la geología de Colombia: Una visión a 2015. Servicio Geológico Colombiano, Publicaciones Geológicas Especiales 33. Bogotá. https://www2.sgc.gov.co/ProgramasDeInvestigacion/Geociencias/Paginas/MapaGeologico.aspx.

(3) Ingeominas (1996). Mapa de zonificación geotécnica de Santafé de Bogotá D. C. proyecto microzonificación sísmica de Santafé de Bogotá D. C. http://seisan.sgc.gov.co/RSNC/Mapa_Zonifica_Geotecnica.pdf.

(4) VIII CPMSIF - PCSMFE. 16 al 21 de agosto de 1987. El suelo de Bogotá y los problemas de cimentaciones. Cartagena, Colombia.

(5) AIS, Asociación Colombiana de Ingeniería (1998). Reglamento Colombiano de Construcción Sismo Resistente NSR-98. http://www.uptc.edu.co/export/sites/default/docentes/oscar_gutierrez/descargas/TxtuloF.pdf.

(6) Ministerio de Ambiente, Vivienda y Desarrollo Territorial de Colombia. (2010). Reglamento Colombiano de Construcción Sismo Resistente NSR-10. http://camacol.co/sites/default/files/secciones_internas/NSR-10_diario_oficial_ 26marzo10.pdf.

(7) Yamin, L. E., Correal, J. F., Reyes, J. C., Ramirez, F., Rincón, J. R., Hurtado, A. I., Dorado, J. F. Sudden collapse of the 27-story "Space" building in Medellin, Colombia. Journal of Performance of Constructed Facilities 2017 (en revisión).

(8) Cano-Marín, R. D., A. Jaramillo-Morilla, F. J. Bernal-Serrano y D. Moreno-Rangel. (2014). Un estudio de caso: rehabilitación singular de edificios de viviendas en la barriada del Parque Alcosa, análisis de daños constructivos comunes y propuesta de intervención. Informes de la Construcción 66, N. ${ }^{\circ}$ 534. http://dx.doi.org/10.3989/ic.12.112.

(9) Anastasopoulos, I. (2013). Structural damage of a 5-storey building: differential settlement due to construction of an adjacent building or because of construction defects? Seventh International Conference on Case Histories in Geotechnical Engineering.

(10) Walhls, H. E. (1981). Tolerable settlement of buildings. Geotechnical and Geoenvironmental Engineering, (107): 1489-1504.

(11) Instituto Nacional de Vías (2013). INV E-128-07. Determinación de la gravedad específica de los suelos y de la llenante minera.

(12) Norma ASTM D7263-09. Standard Test Methods for Laboratory Determination of Density (Unit Weight) of Soil Specimen.

(13) Norma Técnica Colombiana. (2013). Norma NTC 1495. Método de ensayo para determinar en el laboratorio el contenido de agua (humedad) de suelos y rocas, con base en la masa.

(14) Instituto Nacional de Vías. (2013). Norma INV E-122. Determinación en laboratorio del contenido de agua (humedad) de suelo, roca y mezclas de suelo-agregado.

(15) Norma Técnica Colombiana Norma. (1999). NTC 4630. Método de ensayo para la determinación de límite líquido, límite plástico e índice de plasticidad de los suelos cohesivos. 
(16) Instituto Nacional de Vías (2013). Norma INV E-154. Determinación de la resistencia al corte método de corte directo (cd) (consolidado drenado).

(17) Zienkiewicz, O. C., Humpheson, C., Lewis, R. W. (1975). Associated and non-associated viscoplasticity and plasticity in soil mechanics. Geotechnique 25 (4): 671-689.

(18) Yiliang Tu, Xinrong Liu, Zuliang Zhong, Yayong Li (2016). New criteria for defining slope failure using the strength reduction method. Engineering Geology (212): 62-71.

(19) Plaxis 2D (2016). AE Reference Manual. The Netherlands. https://www.plaxis.com/support/manuals/plaxis-2d-manuals/.

(20) Yamin, L., Hurtado, A., Rincón, R., Pulido, J., Reyes, J., Barbat, A. (2014). Evaluation of seismic code specifications using static nonlinear analyses of archetype buildings. Proceedings of the 1oth National Conference in Earthquake Engineering, Earthquake Engineering Research Institute. Anchorage, Alaska.

(21) Burland, J. B; Wroth, C. P. (1975). Settlement of buildings and associated damage. Building Research Establishment. 